\title{
Vertically Integrated Combline Filters in Multilayer PCB
}

\author{
Tinus Stander \\ Carl and Emily Fuchs Institute for Microelectronics, Dept. EEC Engineering \\ University of Pretoria \\ Pretoria, South Africa \\ tinus.stander@up.ac.za
}

\begin{abstract}
Vertical stacking of planar line resonators in multilayer media is an effective footprint reduction technique for high order filters, but strong broadside line coupling often negates the possibility of narrowband filters. It is shown that vertically stacking combline resonators with rotated centerlines leads to moderate bandwidth filters. Four variations of a $5^{\text {th }}$ order filter are demonstrated, achieving 1.92 - $3.3 \mathrm{~dB}$ IL over $10 \% \mathrm{FBW}$ around $10 \mathrm{GHz}$ while occupying between $6.62-22.47$ $\mathrm{mm}^{2}$ surface area on a 7 layer PCB.
\end{abstract}

Keywords-Combline filter, microwave filter, multilayer technology, printed circuits.

\section{INTRODUCTION}

Compact, low-loss filters of moderate and narrow fractional bandwidth (FBW) are required for a variety applications. Vertical stacking resonators [1], often taking the form of interdigital filters [2]-[5], reduces the planar layout size. Thin dielectric layers, however, lead to strong broadside coupling and wide FBW in these filters, even if spread across the planar surface [6]. The problem is further constrained by prescribed or predetermined layer thicknesses, necessitating coupling control using solely lateral geometry definition.

This work explores the use of planar combline resonators [7] in a multilayer PCB stack to realize filters of narrow to moderate $(\sim 10 \%$ FBW $)$ bandwidth. The principles are demonstrated by means of full-wave simulation (CST Microwave Studio 2021).

\section{PRACTICAL COUPLING RANGeS}

Using classical coupled resonator synthesis methods [8], a $5^{\text {th }}$ order all-pole filter of $1 \mathrm{GHz}$ bandwidth centred at 10 $\mathrm{GHz}(10 \% \mathrm{FBW})$, with $-15 \mathrm{~dB}$ pass-band return loss, is found to require inter-resonator couplings of $k_{12}=k_{45}=0.0773$ and $k_{23}=k_{34}=0.0598$ along with $Q_{E 1,2}=12.32$. These values are used to explore the range of practically achievable coupling values in the stack shown in Fig. 1. Five copper signal layers (and two ground layers either side) are created using 3 sheets of 8 mil thickness Mercurywave $9350\left(\varepsilon_{\mathrm{r}}=3.5, \tan \delta=\right.$ $0.0038)$ [9] with $0.5 \mathrm{oz}(17 \mu \mathrm{m})$ copper cladding, interspaced by layers of $1080\left(\varepsilon_{\mathrm{r}}=3.57, \tan \delta=0.0038\right)$ and $2116\left(\varepsilon_{\mathrm{r}}=\right.$ $3.57, \tan \delta=0.0039$ ) prepregs.

\section{A. Interdigital coupling range}

The achievable $k_{23}$ range around $10 \mathrm{GHz}$ (coupling lines on M2 and M3) is first evaluated using an interdigital geometry (Fig. 2(a)) [2] with $L=3.3 \mathrm{~mm}$, varying the dimensions in Fig. 2 as $w \in[0.2,0.5], w_{g} \in[0.2,0.5]$ and horizontal displacement $w_{o} \in[0,2] \mathrm{mm}$. The even and oddmode resonances [8] are found using an Eigenmode solver. The resulting distribution of coupling values in Fig. 3 would indicate a minimum practical coupling of $\sim 0.15$, placing a lower FBW limit of $\sim 25 \%$ on a filter of this prototype function.

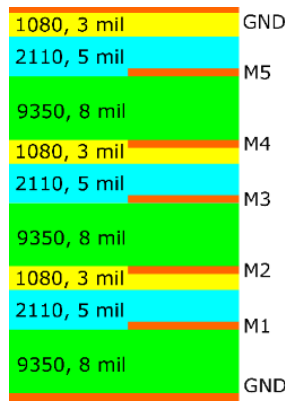

Fig. 1. 5 metal (+2 ground) multi-layer PCB stack

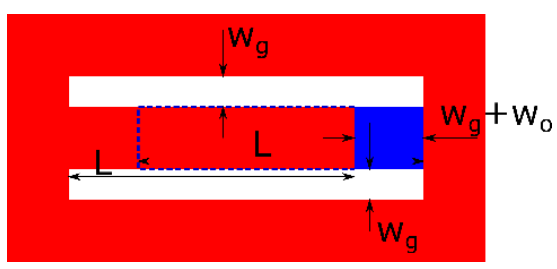

(a)

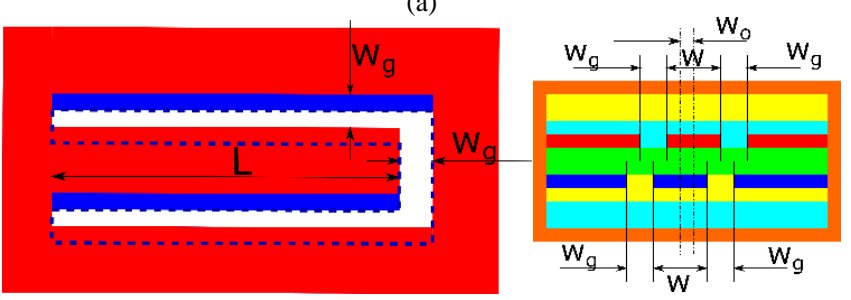

(b)

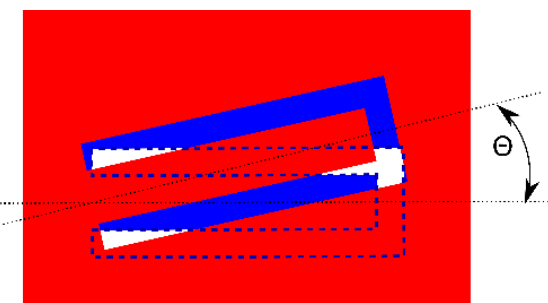

(c)

Fig. 2. Broadside coupling mechanisms. (a) Interdigital, (b) Combline with centreline offset, (c) Combline with centerline rotated.

\section{B. Combline with centreline displaced coupling elements}

Fig. 2(b) shows the coupling of two combline resonators of width $w$ and length $L 3.5 \mathrm{~mm}$ displaced both vertically by the thickness of the 9350 core material, and horizontally by $w_{o}$. Both lines are surrounded by a coplanar ground plane separated by a gap $w_{g}$. Capacitive end-loading may be implemented as shown in e.g. [7], it is omitted here to reduce the board stack complexity and cost. The coupling is varied by adjusting $w \in[0.2,0.5], w_{g} \in[0.2,0.5]$ and $w_{o} \in[0,1]$, with coupling values calculated and plotted in Fig. 5. This topology would lend itself up to FBW $\sim 5 \%$, though the low 
achievable unloaded Q-factor of $~ 115$ would lead to a filter of $\sim 5.5 \mathrm{~dB}$ IL at this FBW. While this is a promising avenue to explore for narrowband filters in low-loss media such as LTCC, or where compact layouts may be augmented with Qenhancement [10], it is not suitable for the current $10 \%$ FBW.

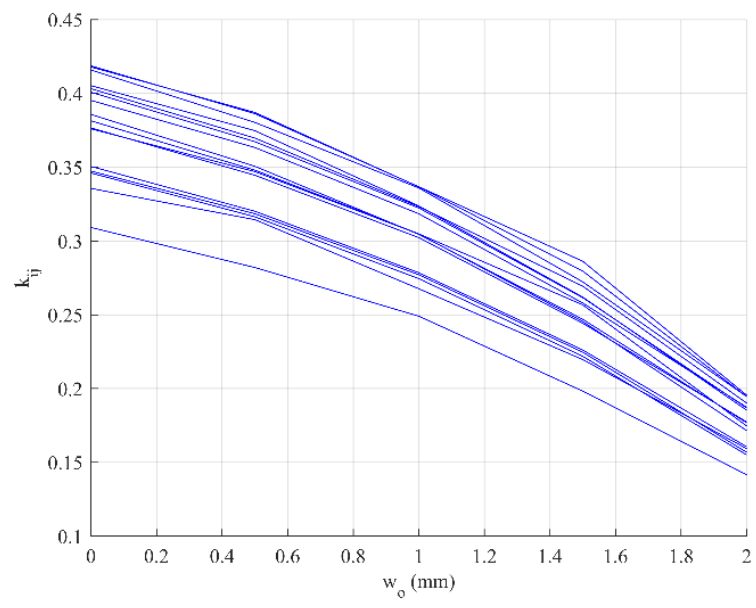

Fig. 3. Coupling range of interdigital elements.

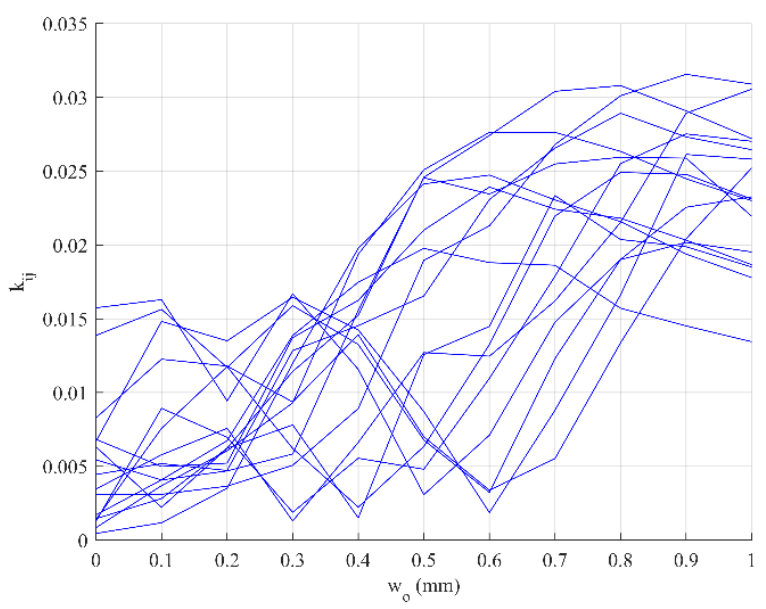

Fig. 4. Coupling range of centreline displaced coupling elements.

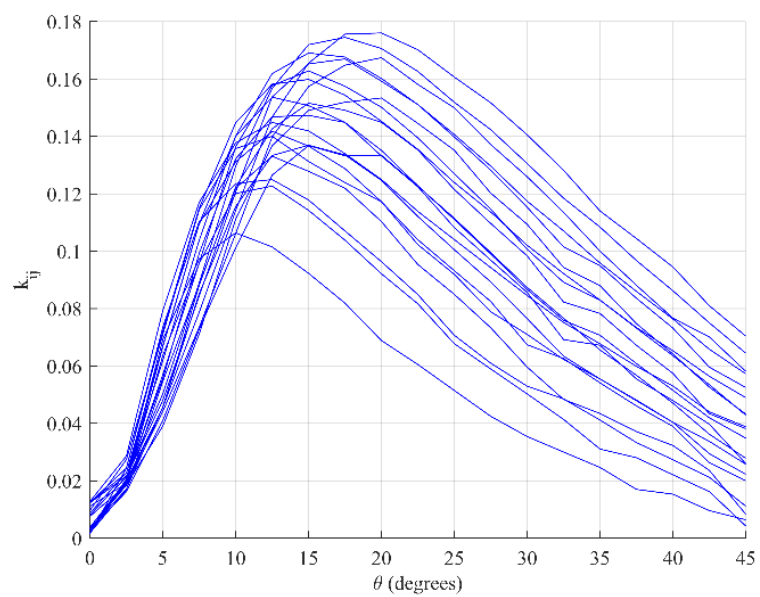

Fig. 5. Coupling range of centreline rotated coupling elements.

\section{Combline with centreline rotated coupling elements}

Maintaining the same line geometry $\left(L, w\right.$ and $w_{g}$ ) from Fig. 2(b), the geometry in Fig. 2(c) varies the coupling between the two combline elements by rotating them by an angle $\theta$ around an axis at the common grounded $x y$ point. By again varying $w \in[0.2,0.5]$ and $w_{g} \in[0.2,0.5]$, with $\theta \in$ $\left[0,45^{\circ}\right]$, the coupling values in Fig. 5 are achieved with even and odd resonances as shown in Fig. 6 . For the given prototype function, this topology would lend itself to implementation of filters of FBW up to $30 \%$, and is implemented here.

\section{FILTER IMPLEMENTATIONS}

Selecting $\theta_{i j}>0$ in all cases leads to a filter shaped like a spiral staircase, while selecting $\theta_{34}$ and $\theta_{45}<0$ (creating counter-clockwise rotation, with the middle element the furthest rotated) creates a more compact layout reminiscent of an antique plough blade. Fig. 5 would further indicate that the same value of $k_{i j}$ may be achieved by using one of two $\theta_{i j}$ values either side of the local maximum, leading to either narrow-rotation or wide-rotation filter implementations. As a result, four variations of the $5^{\text {th }}$ order filter are demonstrated here (Fig. 8), designated "WS" for wide staircase, "NS" for "Narrow Staircase", "WR" for "Wide Plough" and "NR" for "Narrow Plough", each using $w=0.6 \mathrm{~mm}$ and $w_{g}=0.2 \mathrm{~mm}$. The other dimensions in Table I indicate the lengths of the five resonators $L_{1 . . n}$ and the offset angles $\theta_{i j}$. The first and last resonators are use a direct tapped feed, offset by a distance $d_{f}$ from the ground base as shown in Fig. 7, with $w_{f}=0.18 \mathrm{~mm}$ ensuring a $50 \Omega$ transmission line feed.

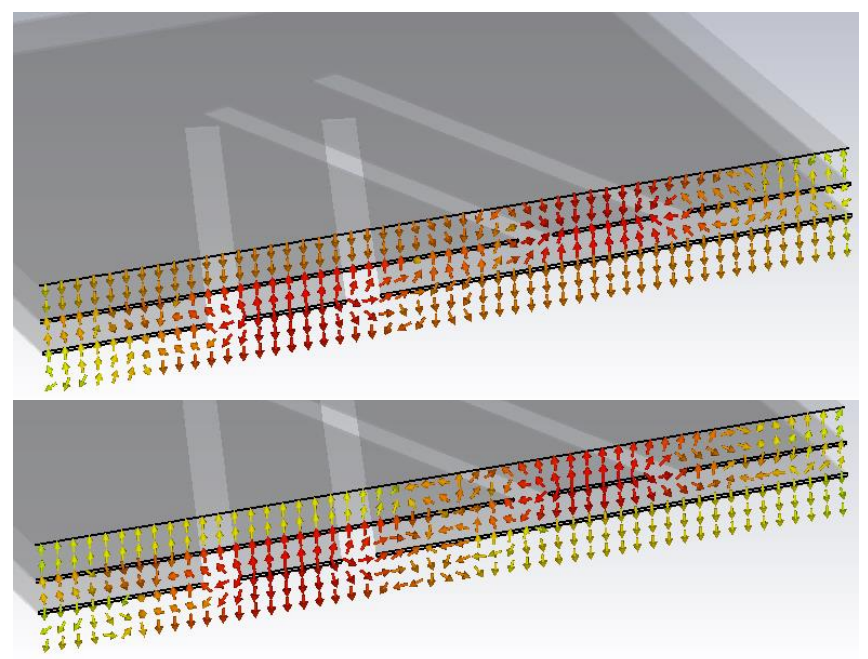

Fig. 6. Even and odd mode resonances for centreline rotated elements.

The results in Fig. 9 would indicate that all four approaches yield feasible filters. The best performing filter is the NS topology with mid-band IL of $1.92 \mathrm{~dB}$, followed by NP $(2.64 \mathrm{~dB})$, WP $(2.8 \mathrm{~dB})$ and WS $(3.3 \mathrm{~dB})$. As expected, NP and NS yield the most compact layouts.

Fig. 9 further indicates that the WS, NS and NP filters all feature asymmetric transmission zeros unaccounted for in the simple all-pole coupling model. This may be due to unintended cross-coupling between elements, or by the presence of non-resonating modes [11]. 
TABLE I. FILTER DIMENSIONS

\begin{tabular}{|c|c|c|c|c|}
\hline & $\boldsymbol{W S}$ & $\boldsymbol{N S}$ & $\boldsymbol{W P}$ & $\boldsymbol{N P}$ \\
\hline$L_{1}(\mathrm{~mm})$ & 3.08 & 3.38 & 3.34 & 3.44 \\
\hline$L_{2}(\mathrm{~mm})$ & 3.02 & 3.34 & 3.27 & 3.37 \\
\hline$L_{3}(\mathrm{~mm})$ & 3.11 & 3.36 & 3.06 & 3.37 \\
\hline$L_{4}(\mathrm{~mm})$ & 3.02 & 3.34 & 3.25 & 3.34 \\
\hline$L_{5}(\mathrm{~mm})$ & 3.19 & 3.39 & 3.45 & 3.43 \\
\hline$\theta_{12}(\mathrm{deg})$ & 27 & 6.45 & 37.5 & 6.40 \\
\hline$\theta_{23}(\mathrm{deg})$ & 36 & 4.60 & 50.5 & 4.60 \\
\hline$\theta_{34}(\mathrm{deg})$ & 37 & 4.50 & 49.5 & 4.40 \\
\hline$\theta_{45}(\mathrm{deg})$ & 28 & 7.25 & 32 & 6.20 \\
\hline$d_{f}(\mathrm{~mm})$ & 0.25 & 0.47 & 0.43 & 0.51 \\
\hline$A\left(\mathrm{~mm}{ }^{2}\right)$ & $\begin{array}{c}6.14 \times 3.66 \\
=22.47\end{array}$ & $\begin{array}{c}3.98 \times 2.54 \\
=10.11\end{array}$ & $\begin{array}{c}3.78 \times 4.21 \\
=15.91\end{array}$ & $\begin{array}{c}3.87 \times 1.71 \\
=6.62\end{array}$ \\
\hline
\end{tabular}

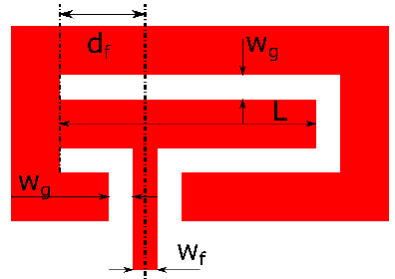

Fig. 7. Coupling range of centreline displaced coupling elements.

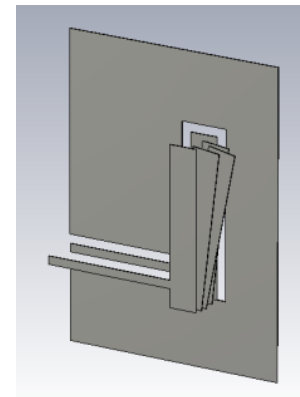

(a)

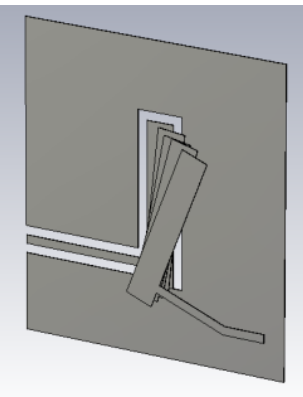

(c)

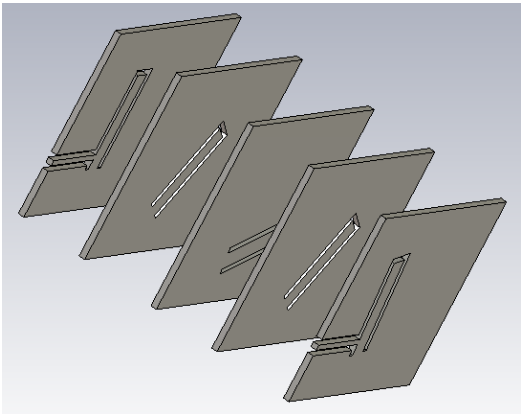

(b)

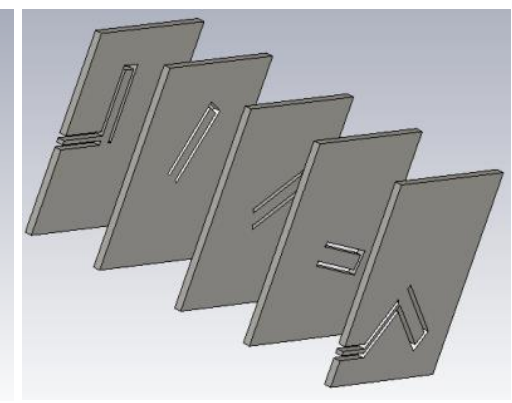

(d)
Fig. 8. Four filter layouts. (a) NP, upper ground planes removed for clarity, (b) WP, exploded view, (c) NS, (d) WS.

\section{CONCLUSION}

Three broadside coupled geometries for vertical integration of planar filters in multi-layer PCB is investigated, with rotationally offset combline elements found to provide suitable coupling values for moderate $10 \%$ FBW filters using typical line geometry. Based on this, four $\mathrm{X}$-band $5^{\text {th }}$ order all-pole filters are demonstrated by simulation, of which the most compact only requires 6.62 $\mathrm{mm}^{2}$ surface area (IL of $2.64 \mathrm{~dB}$ ), while the best performing filter features a minimum in-band IL of only $1.92 \mathrm{~dB}$, at the expense of a larger $10.11 \mathrm{~mm}^{2}$ area.
Future work will improve the synthesis to incorporate the visible asymmetric transmission zeros into the synthesis procedure. Further work may develop a synthesis based on total static capacitance between lines that accounts for the non-uniform per-unit length capacitance of the rotated combline elements. Finally, the results will be validated by prototyping and measurement.

\section{ACKNOWLEDGMENT}

This work was supported by the National Research Foundation of South Africa (NRF) under grant UID114676.

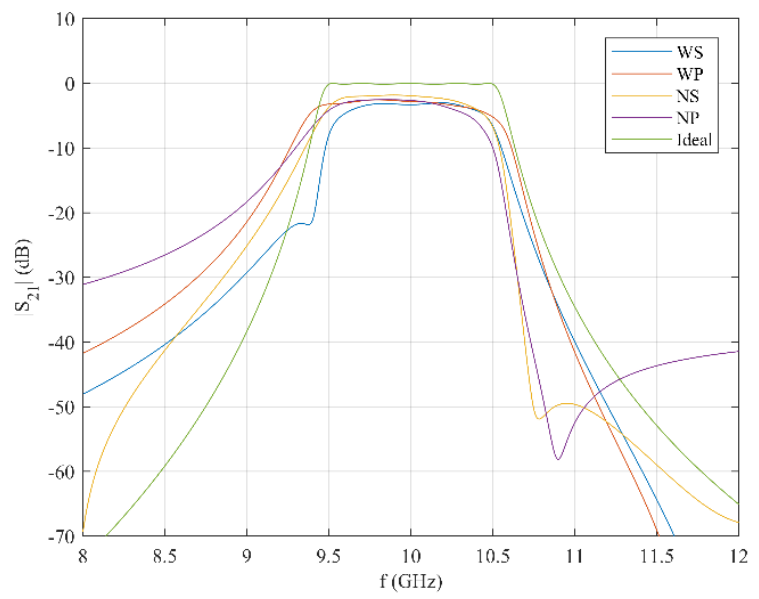

Fig. 9. Comparison of filter transmission responses.

\section{REFERENCES}

[1] Yani Mu, Zhewang Ma, and Deming Xu, “A novel compact interdigital bandpass filter using multilayer cross-coupled folded quarterwavelength resonators," IEEE Microw. Wirel. Components Lett., vol. 15, no. 12, pp. 847-849, Dec. 2005.

[2] A. Perigaud, S. Bila, S. Verdeyme, D. Baillargeat, and D. Kaminsky, "Synthesis of Vertical Interdigital Filters Using Multilayered Technologies," IEEE Trans. Microw. Theory Tech., vol. 60, no. 4, pp. 965-974, Apr. 2012.

[3] A. Perigaud, S. Bila, S. Verdeyme, D. Baillargeat, and D. Kaminsky, "Multilayer Interdigital Structures for Compact Bandpass Filters Providing High Selectivity and Wideband Rejections," IEEE Microw. Wirel. Components Lett., vol. 24, no. 2, pp. 93-95, Feb. 2014.

[4] A. Perigaud, S. Bila, S. Verdeyme, D. Baillargeat, and D. Kaminsky, "Multilayered Coupled Interdigital Resonator Filters for General Chebyshev Filtering Functions," IEEE Trans. Microw. Theory Tech., vol. 64, no. 5, pp. 1465-1475, May 2016.

[5] Z. Hao and J. Hong, "Multilayer interdigital ultra-wideband filter," in 2011 IEEE MTT-S International Microwave Symposium, 2011, pp. 14.

[6] Z. Wang, B. Yan, R. Xu, and W. Lin, “A miniature X-band-embedded multilayer band-pass filter using LTCC technology," Microw. Opt. Technol. Lett., vol. 51, no. 7, pp. 1722-1725, Jul. 2009.

[7] S. C. Aksoy, A. I. Cubukcu, and I. Yildiz, "A multilayered combline filter with high harmonic suppression," in 2016 IEEE MTT-S International Microwave Symposium (IMS), 2016, vol. 2016-Augus, pp. 1-3.

[8] R. J. Cameron, R. R. Mansour, and C. M. Kudsia, Microwave Filters for Communication Systems : Fundamentals, Design and Applications. Hoboken, N.J: Wiley, 2007.

[9] T. Oyedokun, R. H. Geschke, and T. Stander, "Broadband mm-wave propagation characterization of planar groove gap waveguide," Microw. Opt. Technol. Lett., vol. 62, no. 5, pp. 1871-1875, May 2020.

[10] N. Singh and T. Stander, "E-Band Active Q-Enhanced Pseudocombline Resonator in $130 \mathrm{~nm}$ SiGe BiCMOS," J. Infrared, Millimeter, Terahertz Waves, vol. 39, no. 10, pp. 949-953, Oct. 2018.

[11] S. Bastioli and R. V. Snyder, "Nonresonating Modes Do It Better!: Exploiting Additional Modes in Conjunction With Operating Modes to Design Better Quality Filters," IEEE Microw. Mag., vol. 22, no. 1, pp. 20-45, Jan. 2021. 